\title{
Motion compensation of short-range, wide-beam synthetic aperture radar
}

\author{
T. Reichthalhammer and E. Biebl
}

Fachgebiet Höchstfrequenztechnik, Technische Universität München, Munich, Germany

\begin{abstract}
Up to now, SAR systems are a well known possibility for long-range detection. Applying them for shortrange applications with wide-beam antennas, of course, does not increase the resolution but the probability to detect hidden targets with an anisotropic radar cross section significantly in comparison to other detection systems. Working with an appropriate wavelength even improves the possiblity to look through natural cover like grass. An application is detecting fawn while pasture mowing. The main issue in such applications is the antenna's motion in range direction as it is carried by cars or traction engines. If motion is not compensated, the phase cannot be reconstructed correctly, the resolution gets poorer and, in worst case, the target even disappears. Conventional methods for motion compensation either fail for wide beam antennas, since for contributions of wide angles the phase reconstruction is incorrect, or is not applicable for realtime data processing, because the processing time due to interpolation or similar steps is very high. We present a method of image reconstruction regarding motion of the antenna as well as wide beamwidth. This method is analyzed concerning processing time in comparison to the conventional image reconstruction. In our system we use a combination of algorithms. There is shown a comparison for different algorithms dependent of the antenna's motion and aperture angle.
\end{abstract}

\section{Correspondence to:}

T. Reichthalhammer

(reichthalhammer@tum.de)

\section{Introduction}

One of the main characteristics of an SAR system is its independence of the distance between the antenna and the targets concerning the resolution in azimuth direction. That means the resolution stays constant with varying distance. Anyhow a short-range SAR system has advantages, first and foremost a wide-beam system (Gerbl, 2007). Such a system can be used for imaging in automotive applications or in farming to detect wild animals. (Reichthalhammer, 2010) The probability of detection increases if the aperture angle becomes larger since the target is illuminated from different angles. The contributions of e.g. a point scatterer are summed up in proper phase relation. The bigger the aperture angle, the more contributions and the better the contrast in the image.

The quality of an SAR image depends on the accuracy of the phase reconstruction of the single signal contributions of different angles. A consequence of a phase error is a picture with degradation in contrast and resolution. To achieve an exact phase rotation one has to know the exact position of the antenna at each recording point. This issue enlarges if the antenna is not carried by a plane but by a vehicle and the ground is bumby. So motion compensation requires an exact detection of the position and an appropriate signal processing algorithm regarding wide beam contributions.

At short-range systems the special focus concerning motion compensation lays on motion compensation in range direction. The velocity in azimuth can be measured easily due to having exact information of the vehicle's current velocity. But the oscillation of the antenna in range direction is high compared to the distance between target and antenna. In addition the wavelength has a great influence on the quality of the picture as shown later. With existing algorithms for motion compensation in range direction, either contributions of wide angles are not compensated correctly, or realtime processing does not allow them.

Published by Copernicus Publications on behalf of the URSI Landesausschuss in der Bundesrepublik Deutschland e.V. 


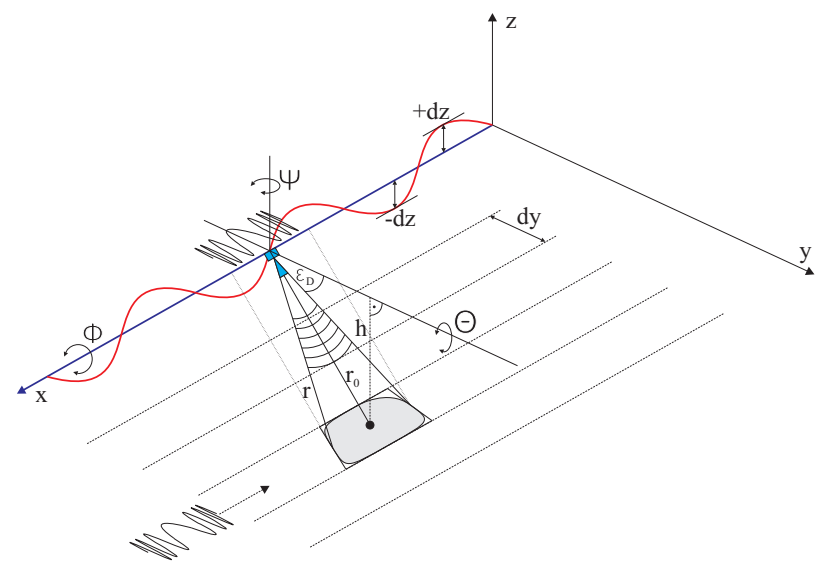

Fig. 1. Coordinate system.

\section{System setup}

Short-range radar imaging with wide-beam antenna is discussed detailed in (Gerbl, 2007). An applicable signal source therefore was developed in (Reichthalhammer, 2010). The crucial point of wide-beam systems is the fast growing doppler bandwidth that postulates a high sample rate. In turn this means a high data volume and a longer processing time. In addition the doppler bandwidth and the processing time is even enlarged if motion has to be compensated. In Fig. 1 the coordinate system of the setup is shown.

The azimuth direction of the system is $\mathrm{x}$, the range direction $r_{0}$ is perpendicular to the azimuth direction. The depression angle $\epsilon_{\mathrm{D}}$ is the angle between the $\mathrm{x}-\mathrm{y}$-plane and the antenna.

\section{SAR imaging}

The general way to reconstruct a recorded scene in azimuth direction is a convolution of the recorded signal with the complex conjugate signal of a point scatterer for each line $\mathrm{d}_{\mathrm{y}}$ of the picture. Our system works in a stop-and-go manner and the doppler frequency caused by the motion of the vehicle can be neglected. So for a stepped frequency system the equation therefore is

$x[l]=\sum_{l=-r_{0} \tan \alpha}^{+r_{0} \tan \alpha} x_{1}[n] \cdot x_{2}[l-n]$,

where $x_{1}$ is the SAR system response and $x_{2}$ the complex conjugate function of $x_{1}$.

Because the illuminated area enlarges with growing distance to the antenna, the compensation function changes with varying distance (Klausing, 2000). The real part of the received signal at a frequency of $24 \mathrm{GHz}$ of a point scatterer at
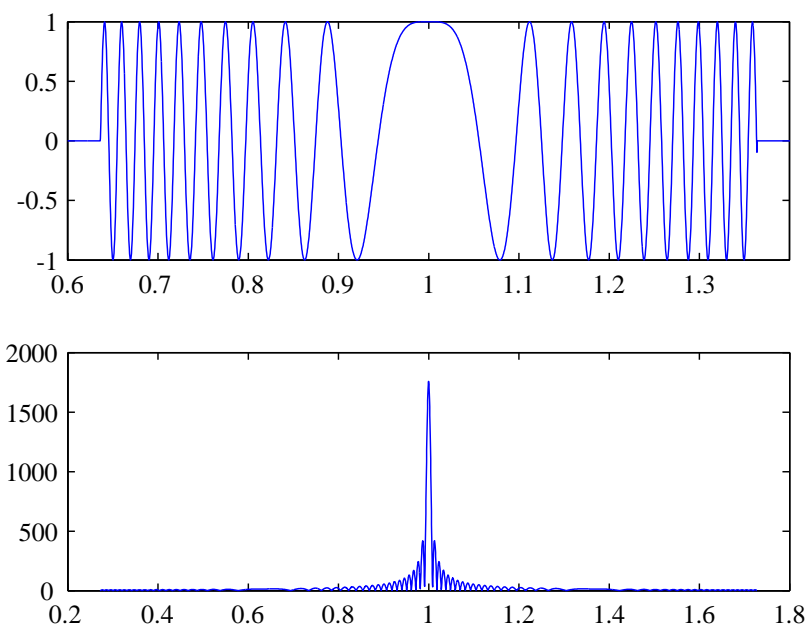

Fig. 2. real part of received signal and magnitude of compressed signal.

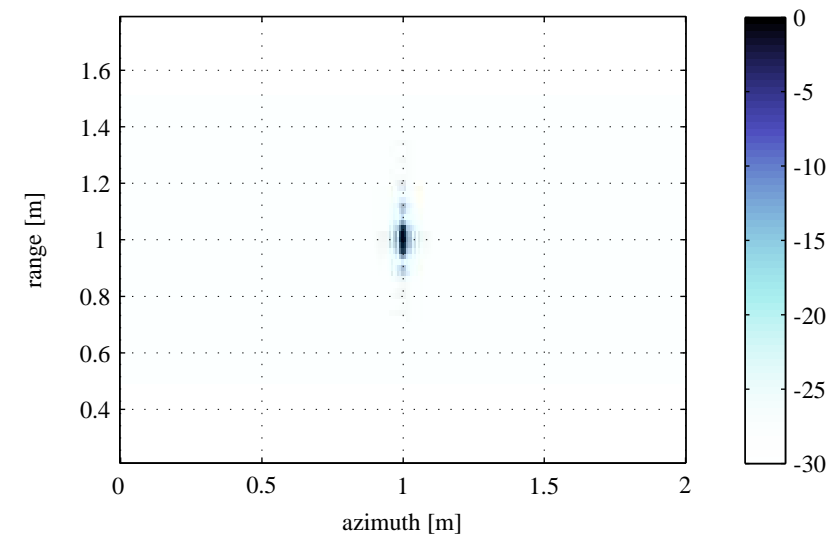

Fig. 3. SAR picture of a point scatterer, $1.8 \mathrm{GHz}$ bandwidth, scaled to its maximum.

a distance $\mathrm{r}_{0}=1 \mathrm{~m}$ and an aperture angle of $20^{\circ}$ is depicted in Fig. 2 assumed constant directivity of the antenna.

The resulting function Fig. 2 is a compression of the spatially extendend received signal with a peak at the position of the target. Obviously the phase velocity increases with increasing angles. The spatial doppler frequency is zero, where the target is located. To get resolution in range equidistant frequency steps inside a given bandwidth are necessary. This results in an unambiguous range which is treated in ( $\mathrm{Re}$ ichthalhammer, 2010). Conventional SAR imaging uses a two dimensional Fourier transformation of the signal $\mathrm{s}(x, t)$ into $\mathrm{s}\left(k_{x}, \omega\right)$ as described e.g. in (Soumekh, 1999). Since our data is sampled equidistantly in azimuth direction the raw data format is $s(x, \omega)$. A typical motionless SAR picture of a point scatterer like the sphere in Fig. 1 is depicted in Fig. 3. 


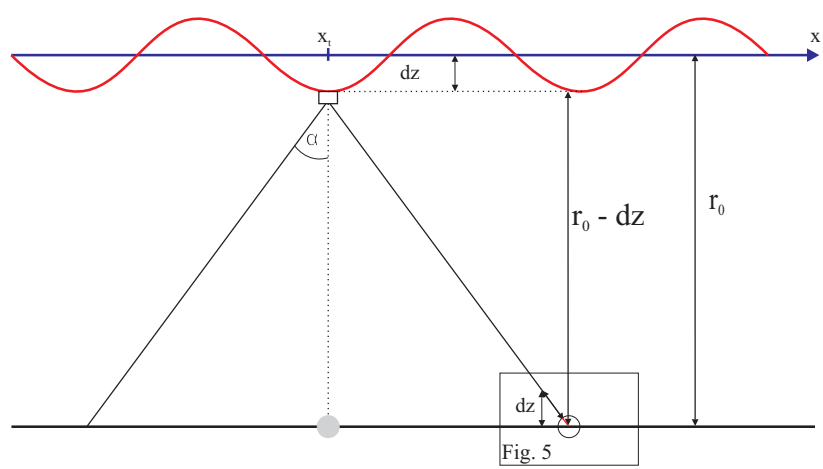

Fig. 4. Occuring compensation error due to antennas movement dependent of aperture angle.

Usually the wavefront curvature cannot be neglected what enforces a transformation of the data from polar into cartesian coordinates. This step can be circumvented if the range stacking algorithm is used (Soumekh, 1999). At range stacking for each given range bin the azimuth compressed signals for each frequency are added what yields the range resolution. This way even for wide beam antennas an interpolation can be avoided and computational time saved.

\section{Motion in short-range SAR systems}

In a 3-dimensional space in principle all 6 degrees of freedom have to be considered. A translational motion in $\mathrm{x}, \mathrm{y}$ and $\mathrm{z}$ direction and a rotatory motion around each axis (pitch, gear, yaw). In our case a few assumptions are done to simplify the problem. Supposed that only a rotation around $\mathrm{x}$ exists along the path $\mathrm{x}$, the target would appear at different positions $\mathrm{y}$ in the antenna's field of view. In worst case -depending on the aperture angle in range and the rotation- the target would even disappear. To compensate this rotation the antenna pattern has to be multiplicated inverse to the image columns. Rotations around y and $\mathrm{z}$ can be neglected, since a huge turn radius of the vehicle and a little curvature of the ground in respect of a short aperture time $T_{S A}$ - the time one target is illuminated by the antenna.

Assumed that the vehicle moves straight ahead the azimuth direction during one synthetic aperture time $T_{S A}$ one can neglect the translational motion in $\mathrm{y}$ as well. As described in (Reichthalhammer, 2010) the system records data along its path $\mathrm{x}$ equidistantly independent of the velocity. Hence a quasi static system is supposed in azimuth direction and motion compensation is not necessary. So the system's movement is reduced to an oscillation in $\mathrm{z}$ and a rotation $\Phi$ around $\mathrm{x}$ which hase to be considered. So the 6-dimensional problem can be reduced to a 2-dimensional problem. For further calculation and simulation even the rotation is neglected.

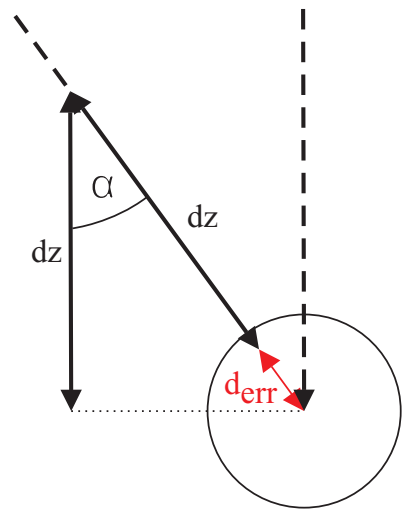

Fig. 5. Magnification Fig. 4.

\section{Motion compensation vs. processing time}

To reconstruct a recorded scene correctly, the main postulation to an SAR system is, that its trajectory has to be known. Simplified a rectilinear trajectory can be assumed, but carried by a vehicle an SAR system cannot follow a straight line. A solution is to detect the motion and rotation and compensate it. For this purpose different algorithms are recommended in literature. The majority of these algorithms try to detect the deviation either by inertial measurement supported by GPS or by analyzing the received signal. Afterwards the received data is manipulated, what means that the phase and the amplitude are corrected. This procedure holds for the case of a small aperture angle and is know as the narrow-beamassumption. For increasing aperture angles these algorithms become defective. The error appears since the reconstruction of the phase is done for each recording point, which corresponds to a column in the SAR picture. If the aperture angle of the antenna becomes bigger each data point can contain information of an object far away from the perpendicular, but a translatory deviation of the antenna always is a term perpendicular to the trajectory. For example the signal of a point scatterer at distance $r$ is

$s=\mathrm{a} \cdot \exp (-2 \mathrm{jkr})$

where $\mathrm{a}$ is an amplitude term and $k=\frac{2 \pi}{\lambda}$ the wavenumber.

With classical compensation like the 2-D Fourier method the singular data point is treated as it only contains information of the scene perpendicular to the trajectory. For example, the distance $\mathrm{r}$ between a sphere and an antenna for $r_{0}=1 \mathrm{~m}$ and $\alpha=20^{\circ}$ degree as depicted in Fig. 4 varies with a deviation of the antenna at $x_{n}$ in $+z$-direction. The magnification of the error made is depicted in Fig. 5. 


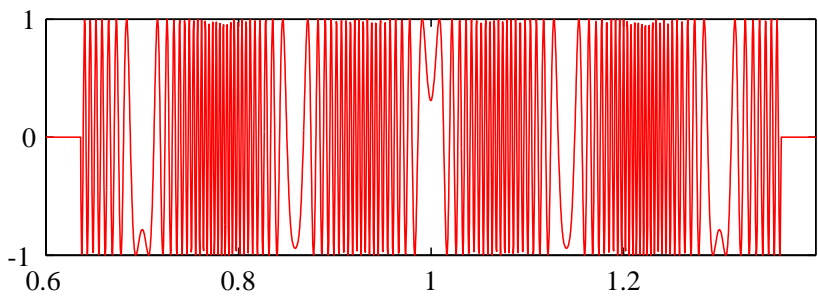

Fig. 6. real part of received signal with motion, $\mathrm{f}=24 \mathrm{GHz}, \alpha=20^{\circ}$, $5 \mathrm{~Hz}$ /aperture, $\mathrm{a}=0.1 \mathrm{~m}, \epsilon_{D}=45^{\circ}$.

To compensate this deviation the data at point $\mathrm{x}_{\mathrm{n}}$ is manipulated by a phase term

$s=\mathrm{a} \cdot \exp (-2 \mathrm{jkr}) \cdot \exp \left(-2 \mathrm{jkd} \mathrm{z}_{\mathrm{z}} \sin \epsilon_{\mathrm{D}}\right)$.

$\mathrm{d}_{\mathrm{z}}$ can be positive or negative. In case of a small beamwidth the variation of $d_{z}$ over the angle can be neglected. Higher angles cause too less phase subtraction in case of motion in $+\mathrm{d}_{\mathrm{z}}$-direction and too much phase addition in case of motion in $-\mathrm{d}_{\mathrm{z}}$-direction.

The absolute error can be defined as

$\mathrm{d}_{\mathrm{err}}=\frac{\left|d_{r}\right|}{\cos \alpha}-\left|d_{r}\right|$

where $\alpha$ is the angle between target and antenna as depicted in Fig. 4 and $\left|d_{r}\right|=\left|d_{z}\right| \sin \epsilon_{D}$.

If an arbitrary harmonic deviation is introduced to the antenna's trajectory with an amplitude of $10 \mathrm{~cm}$ and 5 oscillations per synthetic aperture with $\epsilon_{D}=45^{\circ}$, which is a realistic assumption, the real part of the received signal is depicted in Fig. 6. Hence the typical chirp-like signal form of Fig. 2 is lost. In case of no compensation, the SAR picture of a point scatterer is depicted in Fig. 7.

If compensated with the narrow-beam assumption the signal is filtered as described by Eq. (1) and the picture of Fig. 7 turns to Fig. 8. The maximum of the target decreases, sidelobes appear and the resolution in azimuth decreases significantly, but the processing time itself stays constant.

Note that for using the SAR system for nearly real-time image processing, due to actual processor capabilities the biggest issue is to find a way between sufficient contrast and short processing time. Since there are no smooth transitions between and algorithms mainly focus on one of both sides respectively, one cannot do a compromise.

A well known solution that avoids errors appearing for higher aperture angles is the SAR backprojection algorithm, see (Soumekh, 1999). This algorithm works as follows: SAR data firstly is filtered in range. For each pixel its contribution $s_{d_{r}}$ to the relevant part of the SAR data is reconstructed and vectorial multiplicated with the corresponding SAR data $s_{a z_{n}}$. The sum of the resulting vector yields the reflectivity

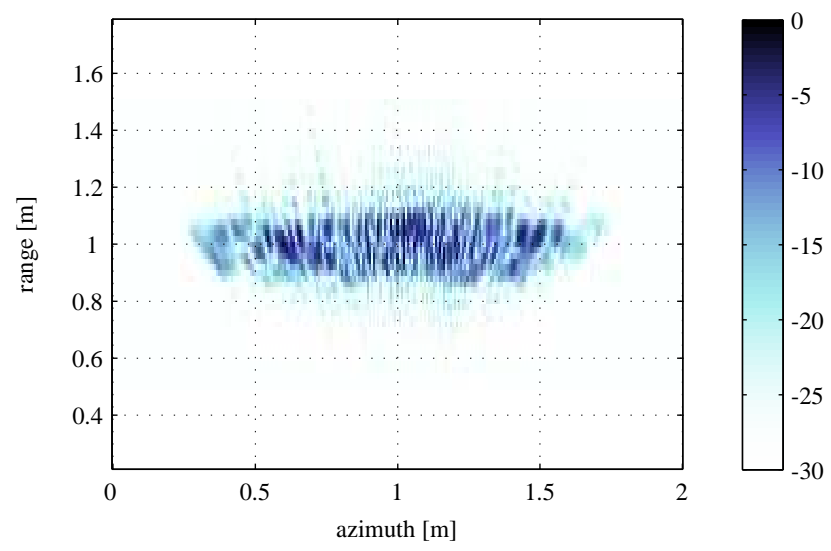

Fig. 7. SAR picture of a point scatterer, $1.8 \mathrm{GHz}$ bandwidth, scaled to its maximum, uncompensated motion.

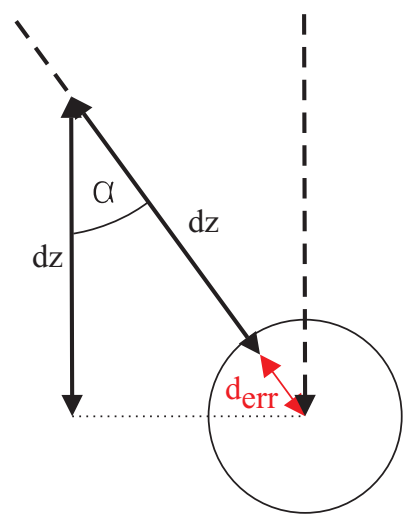

Fig. 8. SAR picture of a point scatterer, $1.8 \mathrm{GHz}$ bandwidth, scaled to its maximum, conventional motion compensation.

of the pixel. This way even a translational motion of the antenna can be compensated, but note that the data have to be filtered in range before, what does not fit to the range stacking algorithm, where an azimuth compression of the data in the first step is compellent. Moreover this algorithm causes a high processor load as treated later. The equation for backprojection is

$x[n]=\sum_{l=n-\left(r_{0}-d_{z}\right) \tan \alpha}^{n+\left(r_{0}-d_{z}\right) \tan \alpha} s_{a z_{n}}[l] \cdot s_{d_{r}}[l]$,

where $x_{d_{r}}$ is the signal different at each point $n$.

A combination of range stacking algorithm and back projection algorithm is an effective way of combining both high quality image reconstruction and acceptable processing time, but as treated above for range stacking the data firstly has to be compensated in azimuth. 


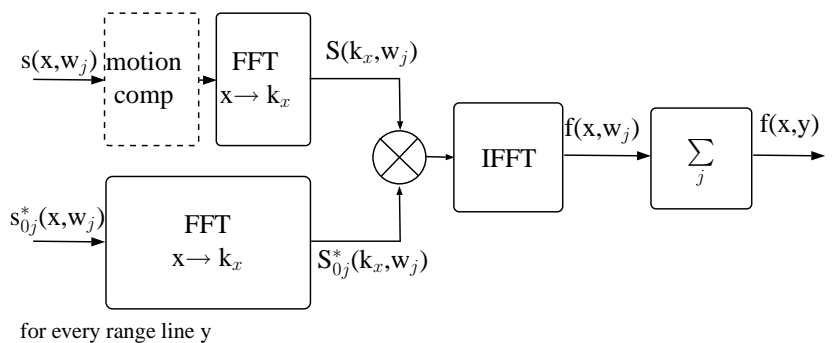

Fig. 9. SAR signal processing with and without motion compensation with narrow beam assumption.

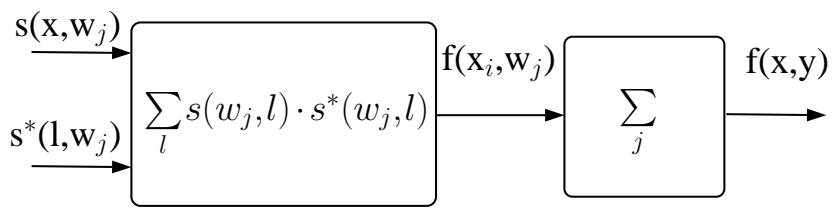

for every range line y

Fig. 10. SAR signal processing with combination of range stacking and backprojection algorithm.

Furthermore the interpolation step of the backprojection algorithm in azimuth direction does not have to be done with our system since the image resolution in azimuth and so $r_{i j}$ inherently are defined by the sampling space $\Delta x$.

In Fig. 9 our processing of the SAR signal is shown with and without motion compensation (dashed box) with the narrow beam assumption, which leads to an image as shown in Fig. 8. The processing has to be done for each range line $y$. To achieve range resolution with range stacking the results for different frequencies are calculated and added each time.

In Fig. 10 the processing is shown if wide beams and other image degrading effects have to be considered. One can see, that nothing of the 2-D Fourier algorithm is left. In both dimensions the processing is substituted by a summation. For each range line, each pixel is processed concerning its reflectivity, as described above. Each range line has to be processed for each frequency. The results for one range line are summed up vectorialy. This way, the picture is reconstructed like the one depicted in Fig. 11.

A comparison for one range line $\mathrm{d}_{r}=1 \mathrm{~m}$ is made in Fig. 12 . In the upper figure the reconstruction of a point scatterer without motion is depicted in comparison to the processing result if a typical motion is not compensated. If not compensated, the image contains no information as depicted in Fig. 7. In the lower figure the results are shown for both, a compensation as described in Fig. 9 and a compensation as treated in Fig. 10.

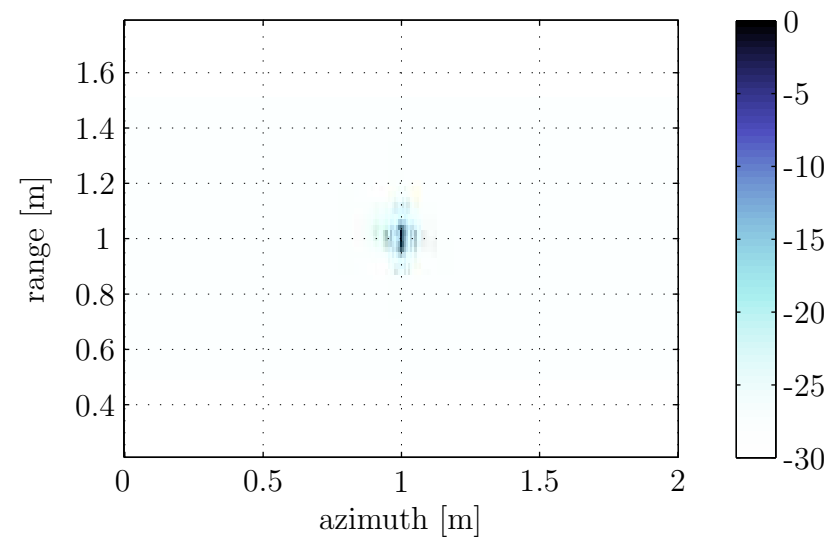

Fig. 11. SAR picture of a point scatterer, $1.8 \mathrm{GHz}$ bandwidth, scaled to its maximum, motion compensation with derivative of backprojection algorithm.
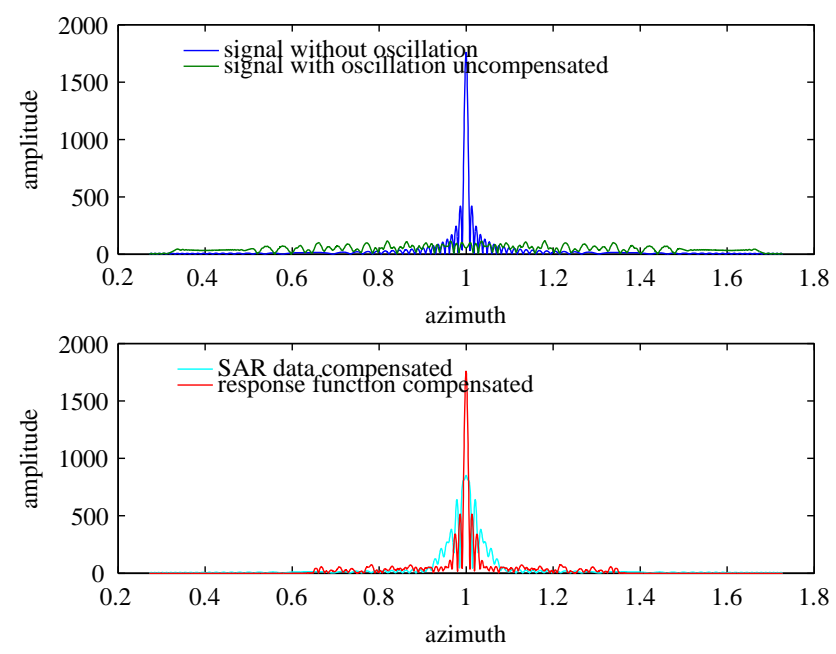

Fig. 12. comparison of SAR signal reconstruction at one range line for no motion, motion without compensation, motion with different compensation methods.

\section{Processing time}

An estimation of the processing time can be done. Therefore a reference processing time have to be defined. In our case this is the processing time without motion compensation as depicted in Fig. 9, that can be described as a 1-D Fourier algorithm with range stacking.

Let $y$ be the number of lines we want to process, $x$ the number of data points in azimuth and $\mathrm{f}$ the number of frequencies used. Than the processing load $O$ without motion compensation is 


$$
\begin{aligned}
& O=y[\underbrace{x \cdot \log _{2}(x)}_{\mathrm{FFT}} \cdot f+\underbrace{x \cdot f}_{\text {summation }}]= \\
& =y \cdot x \cdot f\left[\log _{2}(x)+1\right] .
\end{aligned}
$$

If the SAR raw data is manipulated the processing load of Eq. (6) is extended by the term $x$ and reads as

$$
O=y \cdot x \cdot f\left[\log _{2}(x)+1\right]+x .
$$

In the case of motion compensation as depicted in Fig. 10 the processing load $O$ increases to

$$
\begin{aligned}
& O=y \cdot[\underbrace{l \cdot \log _{2}(l) \cdot f \cdot x}_{\mathrm{FFT}}+\underbrace{f \cdot x}_{\text {multiplication }}+\underbrace{x}_{\text {summation }}]= \\
& =y \cdot x \cdot f\left[l+x+\frac{1}{f}\right],
\end{aligned}
$$

where 1 is the number of data points inside a synthetic aperture dependent on the range and the aperture angle. As explained above there is a huge gap between the two ways of motion compensation regarding processing time increasing with the aperture angle. A possible solution for time critical systems where only one target per synthetic aperture is expected is to process the image at first with the fast algorithm and postprocess the parts of the image where an aim is detected with the slow algorithm. But note, that this method fails if more than one target appears inside one synthetic aperture. If this is not guaranteed, one has to decide between image quality and processing time.

\section{Conclusions}

In this paper we dicussed the motion compensation introduced to an SAR system working in short-range with widebeam antennas. We explained the setup and application of our radar system and show the difference between the algorithms charged by a motion compensation term and a new one, which is a derivation of the backprojection algorithm combined with the range stacking algorithm. The gap between high resolution and fast processing is analyzed and a proposal for processing special target scenes made. Simulation results are depicted and processing time is compared to the original algorithm.

\section{References}

Gerbl, F.: Evaluation of wide-beam, short-range synthetic aperture radar imaging, Ph.D. thesis, TU Muenchen, 2007.

Klausing, H. and Holpp, W.: Radar mit realer und sysnthetischer Apertur, 2000.

Soumekh, M.: Synthetic Aperture Radar Signal Processing with MATLAB Algorithms, 1std edn., 1999.

Reichthalhammer, T. and Biebl, E. M.: Specification and Design of a Fast-Tuning Stepped-Frequency Synthesizer for a ShortRange-SAR-System in the K-Band, 2010. 